\title{
Optimization of carbon ion and proton treatment plans using the raster-scanning technique for patients with unresectable pancreatic cancer
}

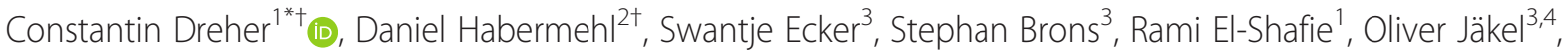
Jürgen Debus ${ }^{1}$ and Stephanie E. Combs ${ }^{2}$

\begin{abstract}
Background: The aim of the thesis is to improve radiation plans of patients with locally advanced, unresectable pancreatic cancer by using carbon ion and proton beams.

Patients and methods: Using the treatment planning system Syngo RT Planning (Siemens, Erlangen, Germany) a total of 50 treatment plans have been created for five patients with the dose schedule $15 \times 3 \mathrm{~Gy}$ (RBE). With reference to the anatomy, five field configurations were considered to be relevant. The plans were analyzed with respect to dose distribution and individual anatomy, and compared using a customized index.

Results: Within the index the three-field configurations yielded the best results, though with a high variety of score points (field setup 5, carbon ion: median 74 (range 48-101)). The maximum dose in the myelon is low (e.g. case 3, carbon ion: $21.5 \mathrm{~Gy}(\mathrm{RBE}))$. A single posterior field generally spares the organs at risk, but the maximum dose in the myelon is high (e.g. case 3, carbon ion: $32.9 \mathrm{~Gy}(\mathrm{RBE})$ ). Two oblique posterior fields resulted in acceptable maximum doses in the myelon (e.g. case 3, carbon ion: $26.9 \mathrm{~Gy}(\mathrm{RBE})$ ). The single-field configuration and the two oblique posterior fields had a small score dispersion (carbon ion: median 66 and 58 (range 62-72 and 40-69)). In cases with topographic proximity of the organs at risk to the target volume, the single-field configuration scored as well as the three-field configurations.
\end{abstract}

Conclusion: In summary, the three-field configurations showed the best dose distributions. A single posterior field seems to be robust and beneficial in case of difficult topographical conditions and topographical proximity of organs at risk to the target volume. A setup with two oblique posterior fields is a reasonable compromise between three-field and single-field configurations.

Keywords: Locally advanced pancreatic cancer, LAPC, Carbon ion, Proton, HIT

\section{Background}

Pancreatic cancer patients are still having a dismal prognosis. About 277,000 people worldwide die each year due to this disease [1]. To date resection is considered to be the only curative treatment. In case of locally advanced unresectable pancreatic cancer (LAPC), neoadjuvant treatment

\footnotetext{
* Correspondence: Constantin.Dreher@stud.uni-heidelberg.de

${ }^{\dagger}$ Equal contributors

'Department of Radiation Oncology, University Hospital of Heidelberg, INF 400, 69120 Heidelberg, Germany

Full list of author information is available at the end of the article
}

approaches including combined chemoradiation with gemcitabine have proven efficacy towards tumor downsizing and lead to a secondary resectability in approximately $30 \%$, even in case of local relapse after primary surgery [2-6]. Modern radiotherapy techniques including IMRT (Intensity-Modulated Radiotherapy) and IGRT (Image-Guided Radiotherapy) offer more conformal dose distributions and thus dose to organs at risk (OAR) can be reduced significantly. Recently it was shown that IMRT treatment in pancreatic cancer patients can provide comparable or less gastrointestinal toxicity than by conventional radiotherapy

\section{Biomed Central}

(C) 2015 Dreher et al. Open Access This article is distributed under the terms of the Creative Commons Attribution 4.0 International License (http://creativecommons.org/licenses/by/4.0/), which permits unrestricted use, distribution, and reproduction in any medium, provided you give appropriate credit to the original author(s) and the source, provide a link to the Creative Commons license, and indicate if changes were made. The Creative Commons Public Domain Dedication waiver (http://creativecommons.org/publicdomain/zero/1.0/) applies to the data made available in this article, unless otherwise stated. 
and therefore contributes to the clinical benefit of the treated patients, especially when used as dose-escalation treatment in combination with IGRT [7, 8]. Charged particle therapy represents an emerging technological advance in oncology. Particle therapy is characterized by an inverted depth-dose-curve, which leads to a low dose deposition within the entry channel and a well-defined high local dose deposition in the Bragg Peak region [9]. The so called Spread Out Bragg Peak (SOBP) allows to irradiate precisely, sparing surrounding normal tissue - both carbon ion and proton radiotherapy have highly conformal dose distributions with high dose deposit in the target volume and an increased sparing of the OARs [9]. High-LET (linear energy transfer) carbon ion beams are characterized by high dose deposition in their trajectory. This results in a high amount of clustered double-strand breaks in the cells'DNA (Deoxyribonucleic acid). So, in contrast to photon and proton beams, carbon ion beams cause an enhanced reduction in clonogenic survival of pancreatic and also of hepatic cell lines [10-13]. Carbon ions in particular offer a higher biological effectiveness due to enhanced and prolonged DNA damaging and induction of bulky lesions, which can be translated into higher RBE (Relative Biological Effectiveness) values $[11,13,14]$. Particle beams are notably appropriate in hepatobiliary and pancreatic malignancies, where radiosensitive normal tissues (e.g. liver, kidneys) are surrounding the target volume [15-17].

There are also encouraging clinical results from Japanese particle therapy facilities, that have conducted small clinical trials and gained experience with carbon ion treatment, using different treatment protocols over the last few years $[16,18]$. Nevertheless, particle therapy of abdominal organs is very complex. Dose application has to be analyzed carefully. That's the reason why the purpose of this study is to evaluate different plan optimization strategies as a preparation for the clinical practice using active raster scanning technology [19]. Different proton and carbon ion field configurations are analyzed with regard to dose distribution and individual anatomy, using a customized rating scheme.

\section{Patients and methods}

\section{Patient characteristics and anatomy criteria}

The medical ethics commission of the medical faculty of Heidelberg consented to this in silico study (S-483/ 2011). Five patients with locally advanced, unresectable pancreatic cancer were included in this study. They were randomly selected from patients treated with standard photon plans at our institution. For treatment planning CT (computed tomography) scans were performed with and without contrast agent and under free breathing. Patients were immobilized in supine position.

Over the cranio-caudal direction of the target volume the minimal distance between two structures was measured in each horizontal slice, and afterwards the mean value was calculated. The mean value depends on variations due to shape and orientation of two structures over the total target volume extension - the following measures were calculated:

1. Mean Xmin kidney ri-le $=$ mean minimum distance between both kidneys

2. Mean Xmin target-kidney le $=$ mean minimum distance between target and left kidney

3. Mean Xmin target-kidney $\mathrm{ri}=$ mean minimum distance between target and right kidney

4. OAR intersection = intersection between target and OARs

Patient characteristics and anatomical criteria are summarized in "Table 1".

\section{Target volume definition}

The treatment planning CT scans with the patients'original volumes of photon irradiation have been transferred to our ion beam treatment planning system. The original target was made up of a PTV (planning target volume) including elective nodal irradiation and a boost volume including the GTV (gross tumor volume) and a margin of $2-4 \mathrm{~mm}$ at the discretion of the responsible

Table 1 Patient characteristics and anatomy criteria

\begin{tabular}{|c|c|c|c|c|c|}
\hline Patient characteristics: & Case1 & Case2 & Case3 & Case4 & Case5 \\
\hline Gender & Male & Male & Female & Male & Male \\
\hline Age at CT scan (years) & 71 & 77 & 64 & 67 & 67 \\
\hline Location & Caput & Caput & Caput & Caput/Corpus & Caput/Corpus \\
\hline Target volume $\left(\mathrm{cm}^{3}\right)$ & 332.63 & 92.31 & 165.75 & 224.39 & 150.35 \\
\hline \multicolumn{6}{|l|}{ Anatomical characteristics: } \\
\hline Mean Xmin kidney ri-le (cm) & 10.1 & 9.6 & 7.5 & 10.6 & 7.5 \\
\hline Mean Xmin target-kidney le $(\mathrm{cm})$ & 5.4 & 6.0 & 3.1 & 5.3 & 4.1 \\
\hline Mean Xmin target-kidney ri $(\mathrm{cm})$ & 3.2 & 3.2 & 3.6 & 2.5 & 2.5 \\
\hline OAR-Intersection & $\begin{array}{l}\text { Large } \\
\text { intestine }\end{array}$ & $\begin{array}{l}\text { Large } \\
\text { intestine }\end{array}$ & $\begin{array}{l}\text { Large intestine, } \\
\text { Stomach/duo-denum, } \\
\text { Liver }\end{array}$ & $\begin{array}{l}\text { Large } \\
\text { intestine }\end{array}$ & $\begin{array}{l}\text { Large intestine, } \\
\text { Stomach/duo-denum, } \\
\text { Liver }\end{array}$ \\
\hline
\end{tabular}


specialist. The boost volumes are defined as the target volumes in the presented cases.

\section{Treatment Planning System (TPS)}

Treatment planning was performed for particle beams using the raster-scanning technique [19]. Treatment planning computation was done by TPS Syngo RT Planning (Siemens, Erlangen, Germany), using the effective dose calculation model as described by Krämer \& Scholz (Local Effect Model, LEM) [20]. Treatment planning with proton beams assumes a fixed RBE value of 1.1. Planning is possible by the use of single field uniform dose optimization (SBO, Single Beam Optimization) or multiple field optimization (IMPT, Intensity Modulated Particle Therapy). Both tools are using intensity modulation, but SBO allows relative weighting factors for each beam. These beams are optimized independently and add up to $100 \%$ of the prescribed dose. IMPT integrates all beams and optimizes simultaneously.

\section{Dose prescription}

At our institution a slightly hypofractioned dose regime has been established for carbon ion irradiation, with a single dose of $3 \mathrm{~Gy}(\mathrm{RBE})$ as described in our forthcoming clinical trial on dose escalated carbon ion therapy for patients with pancreatic cancer [21]. We chose a fraction number of 15 , representing the second escalation dose in the above mentioned PHOENIX trial. The calculated total dose adjusted to the fractionation effect according to the linear-quadratic model and an $\alpha / \beta$-ratio of 2 Gy would result in approximately 56 Gy(RBE) (BED $2 \mathrm{~Gy}(\mathrm{RBE})$ ). This dose remains to our opinion realistic and is effective for both neoadjuvant and definitive treatment.

\section{Field Setup (FS)}

Five different FS at the gantry were considered to be relevant for this study (Table 2). Field configurations including three fields use the SBO tool - to minimize dosimetric uncertainty due to putative anatomical variations the greatest weight was given to the posterior field [22]. These five FS were used for all cases - though slightly adapted to different topography. The gantry beam angles are described according to the International Electrotechnical Commission (IEC).

\section{Treatment plans}

A total of 50 treatment plans were created - taking into account 5 FS, both for carbon ions and protons. The constraints for optimization were put on the same level. For treatment evaluation the original structure sets of photon irradiation have been integrated in our TPS, which is why the OAR duodenum is not available, but integrated in the OAR stomach/ $d d$.

The criteria and the index for comparison are summarized in "Table 3". In general, the DVH (Dose-Volume-Histogram)-parameters were geared to those constraints, which are significant for toxicity and derived from daily clinical practice. As critical tolerance doses were not exceeded, we decided to take lower tolerance limits to better distinguish the different plans. The OAR criteria of the myelon, the kidneys and the liver are of equal value; stomach/dd and large intestine 33 \% less; skin $66 \%$ less. So, in comparison to stomach/dd etc., the criteria value of myelon, kidneys and liver is increased. This distinction is due to the field configurations, for which these organs are consequently exposed to higher doses. The plans were compared with each other by using a specific customized score - the index allocates points amongst the different plans as follows:

Five FS were compared per case and per radiation modality (carbon ions, protons). If a plan does not meet the criteria, it does not receive any points for this criterion. Among the plans meeting the criteria, the plan achieving the best value receives the maximum number of points. The other plans receive fewer points in linear intervals (point grading of the only myelon criterion: 15-12-9-6-3). The cumulative criteria are made up of both target criteria and OAR criteria. The target criteria represent $32 \%$ of the score. The OAR criteria get the remaining $68 \%$ - the relative distribution is identical to the OAR criteria.

\section{Results}

DVH-parameters for proton treatment plans

FS 5 (Fig. 1) has a high dose coverage of the target with V44 $=98.7 \%$ and $98.6 \%$ for case 3 and 4 . The maximum dose $\left(D_{\max }\right)$ in the myelon, and dose criteria for both kidneys are met in both cases. DVHparameters with FS 4 are similar to those with FS 5.

With regard to FS 2 and 3, the target criteria are satisfied in both cases. $D_{\max }$ in the myelon is satisfied in case 3, but narrowly missed in case 4 by both FS (FS 2 and 3: $25.2 \mathrm{~Gy}(\mathrm{RBE})$ and $27.3 \mathrm{~Gy}(\mathrm{RBE})$ ). In case 3 and 4 FS 3 leads to an increased dose deposition in both kidneys. In contrast to that, FS 2 is fulfilling the criteria.

FS 1 (Fig. 1) reaches a high V44 in the target: $97.5 \%$ and $98.1 \%$ (case 3 and 4 ). The dose criterion for the myelon cannot be met (case 3 and 4: 32.4 $\mathrm{Gy}(\mathrm{RBE})$ and 34.0 $\mathrm{Gy}(\mathrm{RBE})$ ), but those for both kidneys can. The mean dose $\left(D_{\text {mean }}\right)$ in the right kidney is only half of the dose with FS 5. 
DVH-parameters for carbon ion treatment plans (Fig. 2)

FS 5 (Fig. 2) meets the target criterion V44: 95.8 and $96.2 \%$ for case 3 and $4 . D_{\max }$ in the myelon is $21.5 \mathrm{~Gy}(\mathrm{RBE})$ and $23.2 \mathrm{~Gy}(\mathrm{RBE})$ in case 3 and 4. For both cases, the kidney criteria are satisfied. DVHparameters of FS 4 are similar to those of FS 5 for both cases.

In case 3 FS 3 does not meet the target criterion and the kidney criteria - but it does in case 4. With regard to $\mathrm{D}_{\max }$ in the myelon, it is the other way around. FS 2 meets with the target criterion V44 and kidney criteria in both cases (but for the $D_{\max }$ ). $\mathrm{D}_{\max }$ in the myelon is 24.4 $\mathrm{Gy}(\mathrm{RBE})$ and $27 \mathrm{~Gy}(\mathrm{RBE})$ in case 4 and 3.

FS 1 (Fig. 2) meets the target criterion V44 for both cases (96.8 and $95.1 \%$ in case 3 and 4). For case 3 the target's volume dose is higher than the one with FS 5 . $\mathrm{D}_{\max }$ in the myelon is $32.9 \mathrm{~Gy}(\mathrm{RBE})$ and $34 \mathrm{~Gy}(\mathrm{RBE})$ in case 3 and 4. DVH-parameters in the kidneys $\left(D_{\text {mean }}\right.$ (right and left kidney) $=0.4 \mathrm{~Gy}(\mathrm{RBE})$ and $2.9 \mathrm{~Gy}(\mathrm{RBE})$ ) are low in case 3 . The same is true for case 4 .

\section{Index evaluation (Fig. 3)}

Protons: FS 4 and 5 yielded the highest scores for the OAR criteria. For case 3 FS 1 achieved the same score as the three-field configurations. Carbon ions: FS 5 has a wide score dispersion for the target criteria (Median 14 (range 2-34)) and cumulative criteria (Median 74 (range 48-101)). Regarding OAR criteria, FS 4 and 5 achieved the highest median scores (58 and 62). Nevertheless, score dispersion is the smallest for FS 1 (range 46-50). FS 2 also has a very small score dispersion (range 30-40). On top of that, in case 3 FS 1 gets almost the same score as the

Table 2 Field setups: characteristics

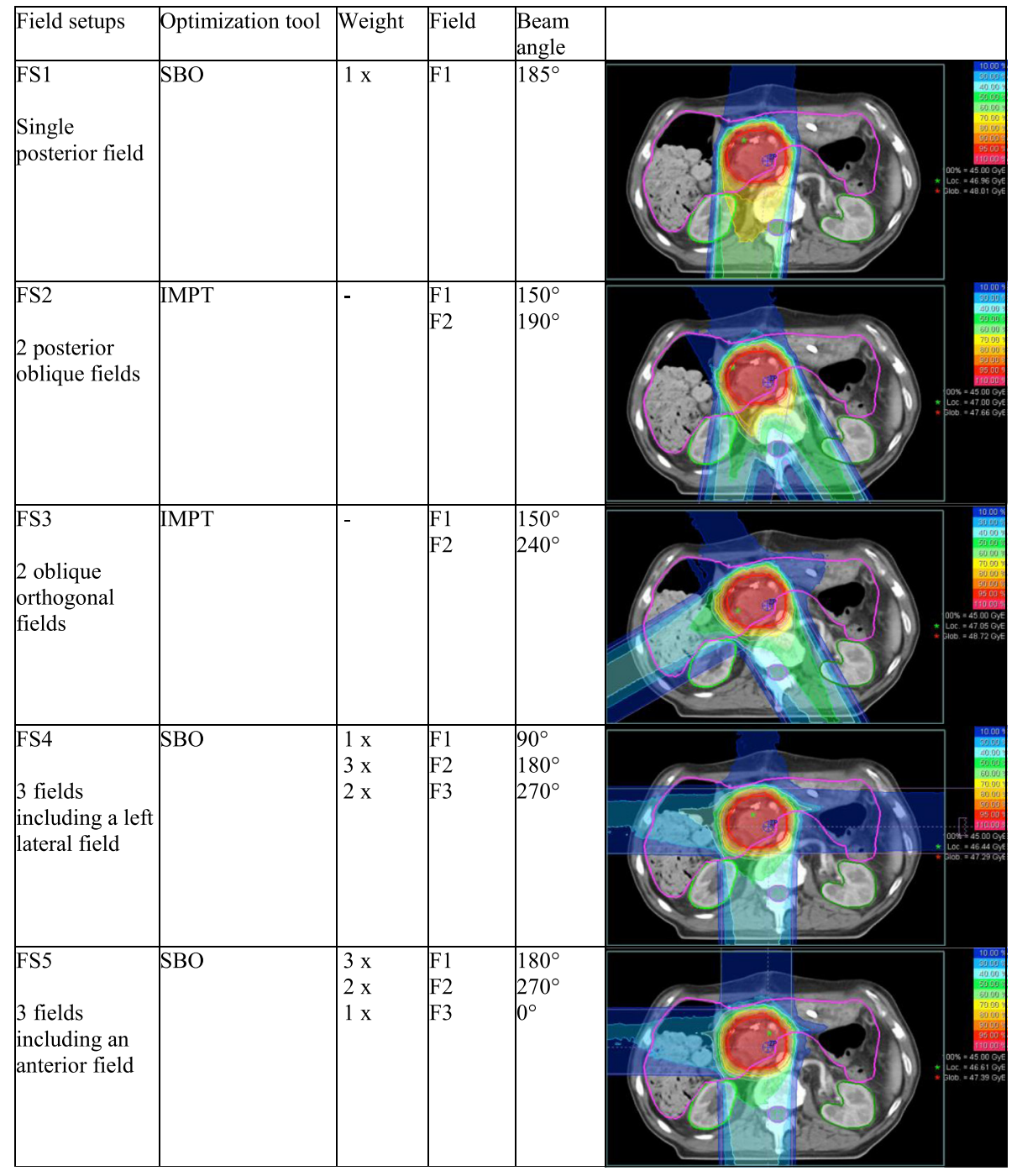


Table 3 Target and OAR criteria

\begin{tabular}{|c|c|c|c|c|c|}
\hline Structure & Constraint & Maximum points & Sum & Score & \\
\hline \multirow[t]{4}{*}{ Target: } & $V 44 \geq 95 \%$ & 10 & \multirow[t]{4}{*}{40} & \multirow[t]{4}{*}{ Target-Criteria } & \multirow{16}{*}{$\begin{array}{l}\text { Cumulative- } \\
\text { Criteria }\end{array}$} \\
\hline & $1-V 42.75<1 \%$ & 10 & & & \\
\hline & Max < 48.15 Gy(RBE) & 10 & & & \\
\hline & Min > 40.00 Gy(RBE) & 10 & & & \\
\hline Myelon: & Max < 24.00 Gy(RBE) & 15 & 15 & OAR-Criteria & \\
\hline \multirow[t]{3}{*}{ Each Kidney: } & V15<15.00\% & 5 & \multirow[t]{3}{*}{15} & & \\
\hline & D25 < 10.00 Gy(RBE) & 5 & & & \\
\hline & Mean < 12.00 Gy(RBE) & 5 & & & \\
\hline \multirow[t]{3}{*}{ Liver: } & V20 < $12.50 \%$ & 5 & \multirow[t]{3}{*}{15} & & \\
\hline & V10<20.00\% & 5 & & & \\
\hline & Mean < 10.00 Gy(RBE) & 5 & & & \\
\hline \multirow[t]{2}{*}{ Stomach/DD: } & Max < 20.00 Gy(RBE) & 5 & \multirow[t]{2}{*}{10} & & \\
\hline & V20<15.00\% & 5 & & & \\
\hline \multirow[t]{2}{*}{ Large intestine: } & Max < 20.00 Gy(RBE) & 5 & \multirow[t]{2}{*}{10} & & \\
\hline & V35 < $10.00 \%$ & 5 & & & \\
\hline Skin: & Max Isodose $<50.00 \%$ & 5 & 5 & & \\
\hline
\end{tabular}

three-field configurations, in case 5 FS 1 even exceeds their score points.

Evaluating the anatomical criteria (Table 1), the cases 3 and 5 clearly offer a topographic proximity of the target to the OARs and the OARs to each other. The mean distance between the right and the left kidney, and the mean distance between the kidneys and the target itself are small in these cases.

\section{Discussion}

Our study focused on treatment planning strategies in patients with unresectable pancreatic cancer. 50 treatment plans for proton and carbon ion beams were evaluated.
We developed a score taking into account target volume coverage and OAR parameters to provide a basis for decision making during the planning process for this patient group. The score demonstrates, that for target volume coverage, all beam angles and combinations provide nearly equivalent scores with a slight advantage for multiple field plans.

In summary, a three-field setup achieved consistently high values throughout the cases. A one-field arrangement with a single posterior field showed in some cases comparable results and overall very little score dispersion. Nevertheless, the maximum doses in the myelon were thoroughly high. But this field configuration seems

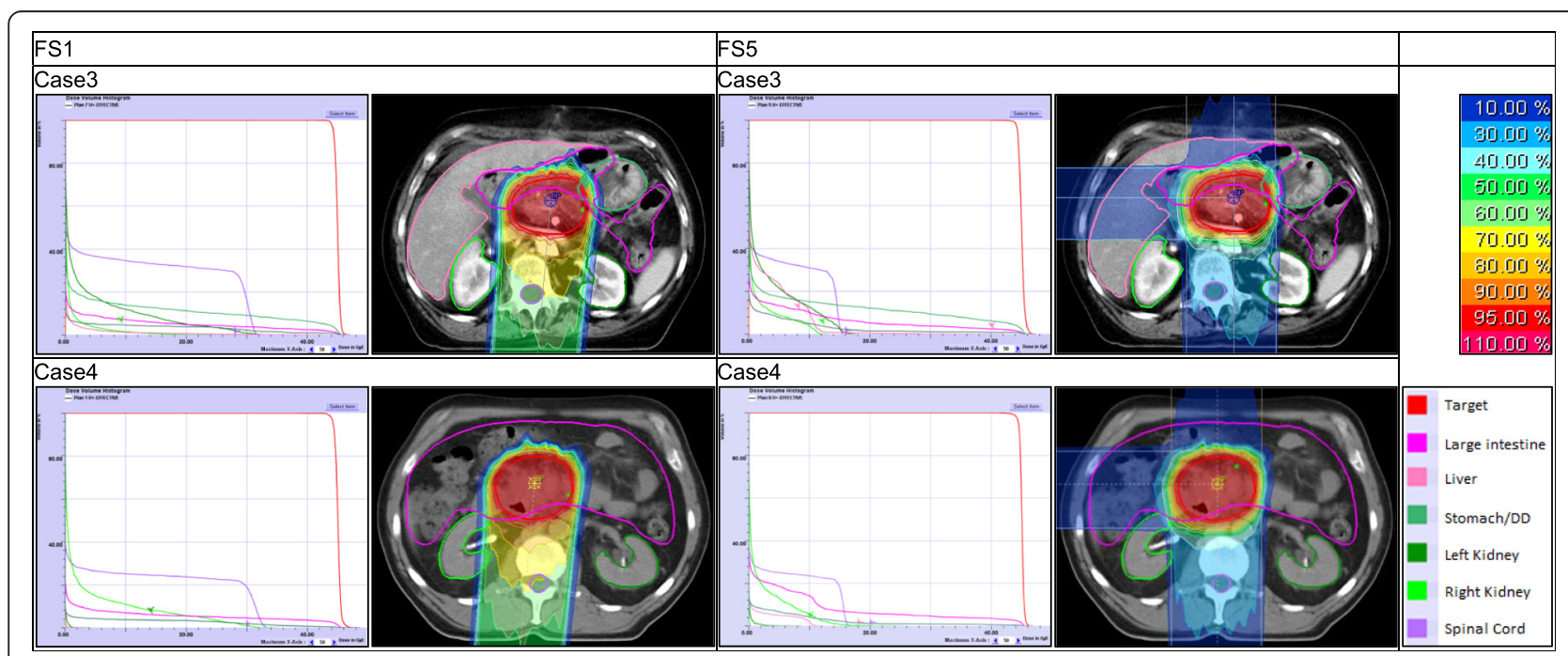

Fig. 1 DVH (Dose-Volume-Histogram) for case 3 and 4 with FS (field setup) 1 and 5: proton 


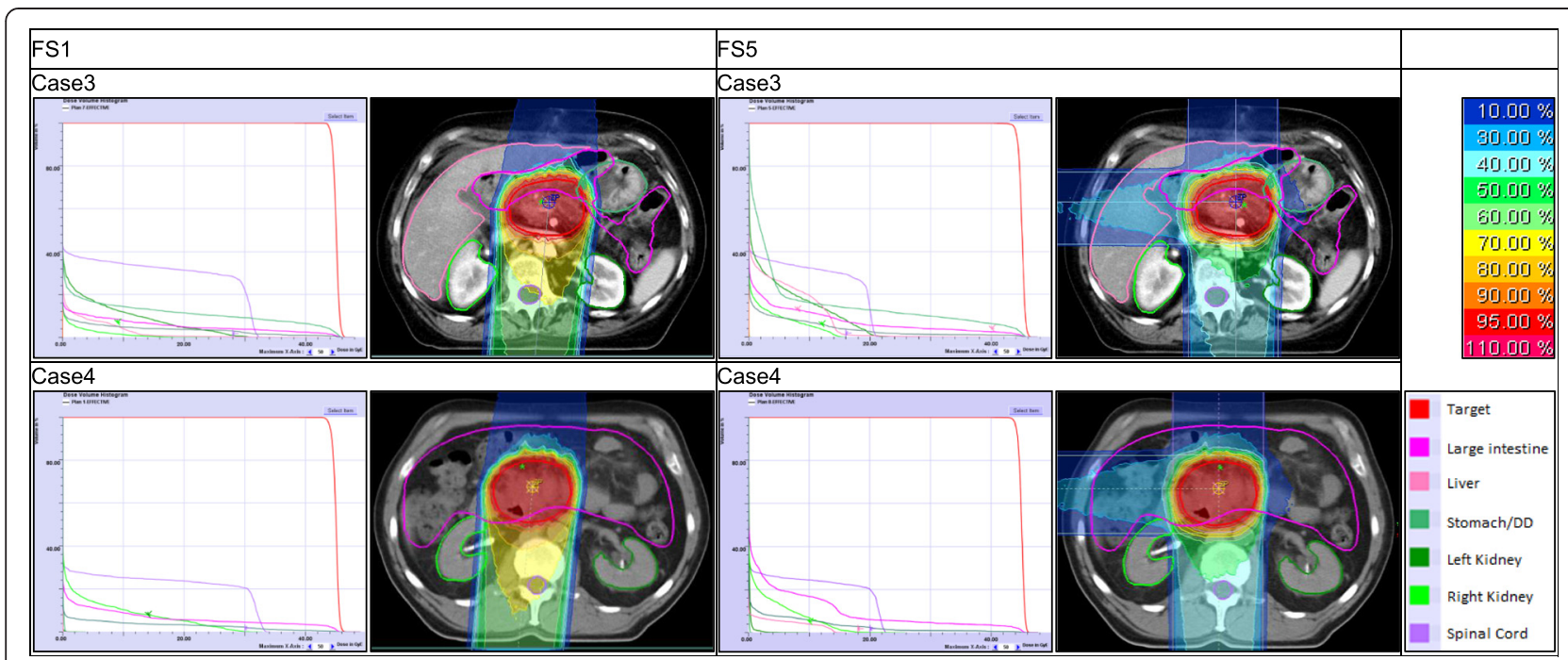

Fig. 2 DVH (Dose-Volume-Histogram) for case 3 and 4 with FS (field setup) 1 and 5: carbon ion

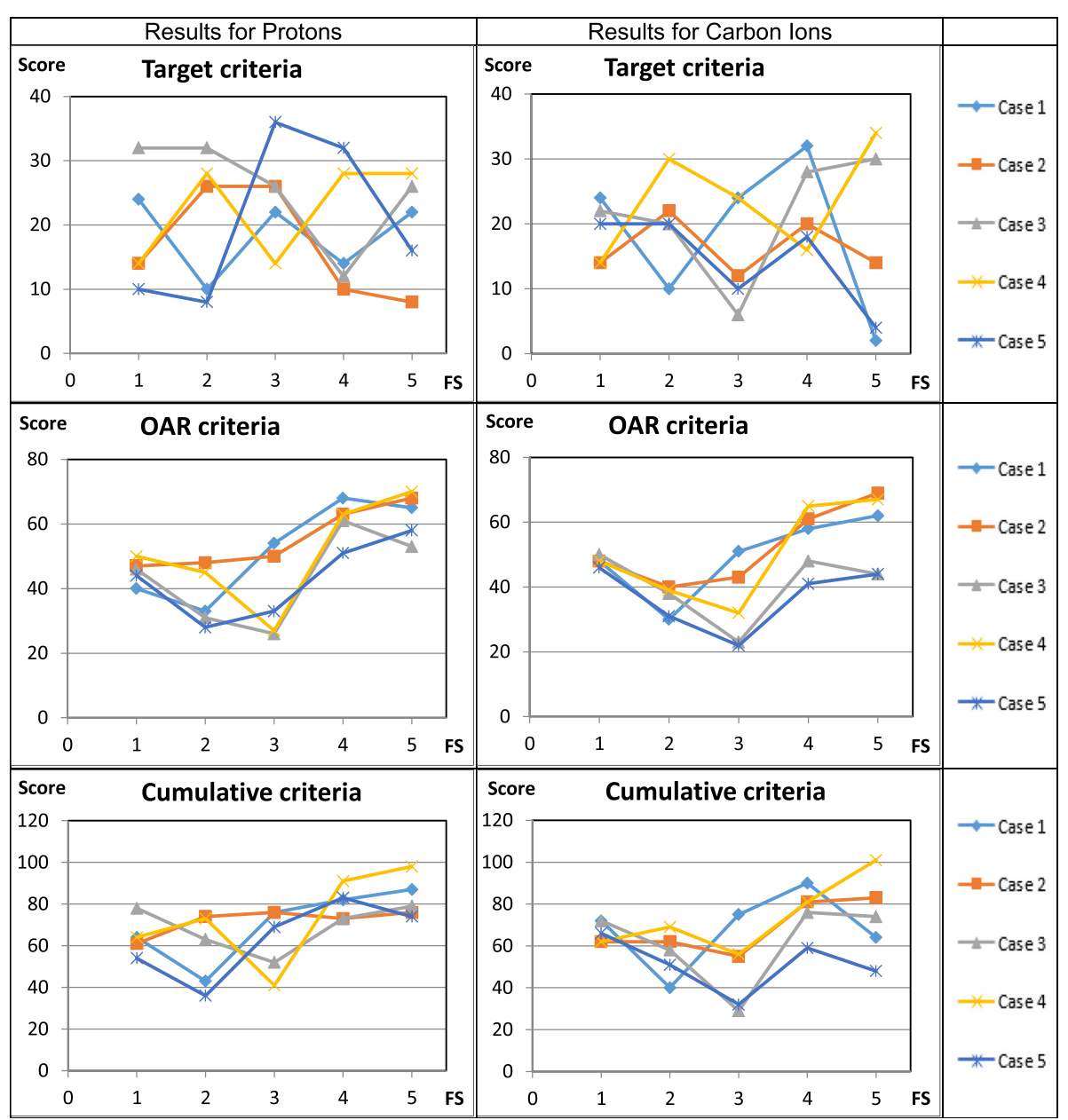

Fig. 3 Index results for proton and carbon ion treatment plans 
to be of advantage in cases of topographic proximity of radiosensitive OARs to each other and to the target volume. The field-setup with two posterior oblique fields through the kidneys showed satisfying results, was able to spare the myelon, and score dispersion was quite small as well.

Particle therapy of abdominal organs is very complex, as inter- and intraindividual changes in organ motion and bowel gas movement may have a serious impact on ion beam dosimetry [22, 23]. Kumagai and colleagues reported a treatment plan analysis of passive scattered carbon ion beams at their facility and found out, that both anterior-posterior and left-right field constellations caused the highest dose affections (mainly because of gastrointestinal gas bubbles) [22]. The three-field configurations include such fields, which is why they have to be evaluated critically. Our results also point to more robustness of a single posterior field compared to threefield arrangements. In this setting beam paths are practically not affected by gas fillings in stomach (left-right) and intestine (anterior-posterior). On top of that, interand intrafractional variations due to breathing may cause relevant changes in the beam path leading to adverse effects. Taniguchi and colleagues further analyzed doses in duodenum and stomach in patients with unresectable pancreatic carcinoma treated with a five-fraction protocol. Results show a decreasing dose to the OARs during expiration compared to inspiration [24]. The interaction of beam and organ motion can cause interplay effects and potentially lead to (unexpected) dose variations in target structures and thus unwanted normal tissue exposure [25]. However, intra- and inter-fractional changes are described but not totally understood, which is why we need re-planning scenarios, especially in scanned ion beam treatment, where slight changes may result in significant dose variations [22-24, 26]. In general, we are referring to Japanese experiences in ion beam therapy of LAPC. But one has to be clear about the fact, that irradiation was performed by the use of scattered ion beam therapy. Our department made investigations in robustness in scanned ion beam therapy, which we can rely on. Batista et al. has presented data about pancreatic cancer, that supported our results. A single posterior field and two oblique posterior fields show good results in case of robustness [27]. On top of that Richter et al. has made investigations in the topic of robustness of ion beam irradiation of liver tumors. The group was able to show, that fractionation is a potential tool to reduce dose inhomogenity by interplay effects $[28,29]$. That is one of the reasons why our PHOENIX trial has dose escalation steps starting with 12 fractions [21].

In out treatment plan computations doses to OARs were thoroughly uncritical. But distinct field setups lead to different risk profiles. Whereas posterior fields deposit higher doses to the myelon and partial volumes of the kidneys, right lateral fields are surely affecting partial liver volumes. Higher doses in intestinal structures were found when left lateral and anterior fields were used (three-field configurations). Even though only small subvolumes received remarkable doses, this could theoretically result in clinically relevant complications. The experience on comparable dose protocols with intestinal structures in close proximity to the target volume is based on carbon ion therapy of sacral chordoma and locally recurrent rectal cancer, where no higher gastrointestinal toxicities were recorded [30, 31].

The single posterior field setup is of major concern, because of a steep RBE-increase at the distal end of the SOBP, which could result in unexpected high doses to the small intestine ventrally of the pancreatic tumor. Calculated doses to intestines were in the range of tolerance. With regard to the $\mathrm{DVH}$, the single posterior field can spare most of the OARs. High doses in the myelon were calculated, but did not exceed general QUANTEC (Quantitative Analyses of Normal Tissue Effects in the Clinic) dose constraints [32].

Nevertheless, tolerance doses of the OARs are not well defined for carbon ion irradiation and are extrapolated from photon-based data as long as no new dose-volume thresholds are defined. An important limitation of the LEM-based TPS is also the underlying biological dataset. According to our current clinical practice we do not differentiate the radiobiological characteristics of normal tissue and tumor during treatment plan optimization. Experience with carbon ion beams based on newer biological optimized LEM-algorithms within the TPS are only theoretical and not debated in this work [33].

Topographical influence and advantage of the singlefield configuration is mainly expressed by carbon ion beams. It is likely that the proton beams are at a disadvantage due to their wider lateral dose gradient [9]. Due to this beam broadening, especially with deeper located targets, smearing of the dose distribution possibly results in plans inferior to advanced photons. Basically, a direct comparison between carbon ion and proton beams could be drawn but this may not be useful, as we are currently still working with an earlier version of LEM [34]. Therefore the dose in the proximal part of the beam channel is rather overestimated and the dose in the target itself is underestimated as recently demonstrated by Grun and co-workers with an improved version of the local effect model (LEM IV) [35]. Carbon ion radiation would result in more beneficial dose distributions both in the target and OARs than shown in the presented data.

Nonetheless, our study was able to present possible field setups and evaluated them by the use of a customized score. With reference to our results, particle therapy 
of pancreatic cancer is possible by the use of all 5 field setups. Distinct topographical conditions should be taken into account.

Future work of our group will focus on even more differentiated radiobiological-based treatment planning, which includes different $\alpha / \beta$ ratios for normal and target tissues.

\section{Conclusion}

In summary, field configurations with three fields showed the best dose distributions - for both carbon ion beams and proton beams. Nevertheless, three-field configurations are highly influenced by gastrointestinal variations. A single posterior field deposits high doses in the myelon, but seems to be the most robust one and showed good results for difficult and varying topographical conditions. A setup with two oblique posterior fields generated reproducible results and can be set as a reasonable compromise between three fields and one field configurations.

\begin{abstract}
Abbreviations
BED: biologically effective dose; CT: computed tomography; DEGRO: German Radiation Oncology Society; $\mathrm{D}_{\max }$ : maximum dose; $\mathrm{D}_{\text {mean }}$ : mean dose; DNA: Deoxyribonucleic Acid; DVH: Dose-Volume-Histogram; FS: Field Setup; GTV: gross tumor volume; HIT: Heidelberg Ion Beam Therapy Center; IEC: International Electrotechnical Commission; IGRT: Image-Guided Radiotherapy; IMPT: Intensity Modulated Particle Therapy; IMRT: IntensityModulated Radiotherapy; LAPC: locally advanced pancreatic cancer; LEM: Local Effect Model [20]; LET: Linear Energy Transfer; OAR: organ at risk; PTV: planning target volume; QUANTEC: Quantitative Analyses of Normal Tissue Effects in the Clinic; RBE: Relative Biological Effectiveness; SBO: Single Beam Optimization; SOBP: Spread Out Bragg Peak; TPS: Treatment Planning System.
\end{abstract}

\section{Competing interest}

The authors declare that they have no competing interests.

\section{Authors' contributions}

$\mathrm{CD}$ and $\mathrm{DH}$ designed the concept, collected the relevant parameters, performed the analysis, wrote and drafted the manuscript. SE and SB helped designing the concept and contributed to the data collection. RE participated in the design of the study. JD and OJ participated in the design of the study, its coordination and helped to draft the manuscript. SEC designed the concept, participated in the critical analysis and critically revised the manuscript. All authors read and approved the final manuscript.

\section{Acknowledgment}

Parts of the data were presented at the Annual Meeting of the German Radiation Oncology Society (DEGRO) in Berlin, 2013, and the work was honored with the "Best Scientific Talk Award".

\footnotetext{
Author details

'Department of Radiation Oncology, University Hospital of Heidelberg, INF 400, 69120 Heidelberg, Germany. ${ }^{2}$ Department of Radiooncology, Klinikum rechts der Isar, Ismaninger Str. 22, 81675 Munich, Germany. ${ }^{3}$ Heidelberg Ion Beam Therapy Center (HIT), INF 450, 69120 Heidelberg, Germany. ${ }^{4}$ Department of Medical Physics in Radiation Oncology, German Cancer Research Center, INF 280, 69120 Heidelberg, Germany.
}

Received: 3 July 2015 Accepted: 9 November 2015

Published online: 21 November 2015

\section{References}

1. Raimondi S, Maisonneuve P, Lowenfels AB. Epidemiology of pancreatic cancer: an overview. Nat Rev Gastroenterol Hepatol. 2009;6(12):699-708. doi:10.1038/nrgastro.2009.177.

2. Gillen S, Schuster T, Meyer Zum Buschenfelde C, Friess H, Kleeff J. Preoperative/neoadjuvant therapy in pancreatic cancer: a systematic review and meta-analysis of response and resection percentages. PLoS Med. 2010; 7(4):e1000267. doi:10.1371/journal.pmed.1000267.

3. Habermehl D, Kessel K, Welzel T, Hof H, Abdollahi A, Bergmann F, et al. Neoadjuvant chemoradiation with gemcitabine for locally advanced pancreatic cancer. Radiat Oncol. 2012;7:28. doi:10.1186/1748-717X-7-28.

4. Naumann P, Habermehl D, Welzel T, Debus J, Combs SE. Outcome after neoadjuvant chemoradiation and correlation with nutritional status in patients with locally advanced pancreatic cancer. Strahlenther Onkol. 2013. 189(9):745-52. doi:10.1007/s00066-013-0393-3.

5. Habermehl D, Brecht IC, Bergmann F, Welzel T, Rieken S, Werner J, et al. Chemoradiation in patients with isolated recurrent pancreatic cancer - therapeutical efficacy and probability of re-resection. Radiat Oncol. 2013;8:27. doi:10.1186/1748-717X-8-27.

6. Nakamura A, Itasaka S, Takaori K, Kawaguchi Y, Shibuya K, Yoshimura M, et al. Radiotherapy for patients with isolated local recurrence of primary resected pancreatic cancer. Prolonged disease-free interval associated with favorable prognosis. Strahlenther Onkol. 2014;190(5):485-90.

7. Yovino S, Poppe M, Jabbour S, David V, Garofalo M, Pandya N, et al. Intensity-modulated radiation therapy significantly improves acute gastrointestinal toxicity in pancreatic and ampullary cancers. Int J Radiat Oncol Biol Phys. 2011;79(1):158-62. doi:10.1016/j.jijobp.2009.10.043.

8. Combs SE, Habermehl D, Kessel K, Bergmann F, Werner J, Brecht I, et al. Intensity modulated radiotherapy as neoadjuvant chemoradiation for the treatment of patients with locally advanced pancreatic cancer : Outcome analysis and comparison with a 3D-treated patient cohort. Strahlenther Onkol. 2013;189(9):738-44. doi:10.1007/s00066-013-0391-5.

9. Suit H, DeLaney T, Goldberg S, Paganetti H, Clasie B, Gerweck L, et al. Proton vs carbon ion beams in the definitive radiation treatment of cancer patients. Radiother Oncol. 2010;95(1):3-22. doi:10.1016/j.radonc.2010.01.015

10. Prescribing, recording, and reporting proton-beam therapy. International Commission on Radiation Units and Measurements2007 December 1, 2007. doi: 10.1093/jicru/ndm021

11. El Shafie RA, Habermehl D, Rieken S, Mairani A, Orschiedt L, Brons S, et al. In vitro evaluation of photon and raster-scanned carbon ion radiotherapy in combination with gemcitabine in pancreatic cancer cell lines. J Radiat Res. 2013:54 Suppl 1:i113-9. doi:10.1093/jrr/rrt052.

12. Georgakilas AG, O'Neill P, Stewart RD. Induction and repair of clustered DNA lesions: what do we know so far? Radiat Res. 2013;180(1):100-9. doi:10.1667/ RR3041.1.

13. Habermehl D, llicic K, Dehne S, Rieken S, Orschiedt L, Brons S, et al. The relative biological effectiveness for carbon and oxygen ion beams using the raster-scanning technique in hepatocellular carcinoma cell lines. PLOS ONE. 2014;9(12):e113591. doi:10.1371/journal.pone.0113591.

14. Oonishi K, Cui X, Hirakawa H, Fujimori A, Kamijo T, Yamada S, et al. Different effects of carbon ion beams and X-rays on clonogenic survival and DNA repair in human pancreatic cancer stem-like cells. Radiother Oncol. 2012; 105(2):258-65. doi:10.1016/j.radonc.2012.08.009.

15. Habermehl D, Debus J, Ganten T, Ganten MK, Bauer J, Brecht IC, et al. Hypofractionated carbon ion therapy delivered with scanned ion beams for patients with hepatocellular carcinoma - feasibility and clinical response. Radiat Oncol. 2013;8:59. doi:10.1186/1748-717X-8-59.

16. Shinoto M, Yamada S, Yasuda S, Imada H, Shioyama Y, Honda H, et al. Phase 1 trial of preoperative, short-course carbon-ion radiotherapy for patients with resectable pancreatic cancer. Cancer. 2013;119(1):45-51. doi:10.1002/ cncr.27723.

17. Schneider RA, Vitolo V, Albertini F, Koch T, Ares C, Lomax A, et al. Small bowel toxicity after high dose spot scanning-based proton beam therapy for paraspinal/retroperitoneal neoplasms. Strahlenther Onkol. 2013;189(12): 1020-5. doi:10.1007/s00066-013-0432-0.

18. Okada T, Kamada T, Tsuji H, Mizoe JE, Baba M, Kato S, et al. Carbon ion radiotherapy: clinical experiences at National Institute of Radiological Science (NIRS). J Radiat Res. 2010;51(4):355-64.

19. Haberer T, Becher W, Schardt D, Kraft G. Magnetic scanning system for heavy ion therapy. Nuclear Instruments and Methods in Physics Research Section A: Accelerators, Spectrometers, Detectors and Associated 
Equipment. 1993;330(1-2):296-305. doi:http://dx.doi.org/10.1016/01689002(93)91335-K

20. Kramer M, Scholz M. Treatment planning for heavy-ion radiotherapy: calculation and optimization of biologically effective dose. Phys Med Biol. 2000;45(11):3319-30.

21. Combs SE, Habermehl D, Kieser M, Dreher C, Werner J, Haselmann R, et al. Phase I study evaluating the treatment of patients with locally advanced pancreatic cancer with carbon ion radiotherapy: the PHOENIX-01 trial. BMC Cancer. 2013;13:419. doi:10.1186/1471-2407-13-419.

22. Kumagai M, Hara R, Mori S, Yanagi T, Asakura H, Kishimoto R, et al. Impact of intrafractional bowel gas movement on carbon ion beam dose distribution in pancreatic radiotherapy. Int J Radiat Oncol Biol Phys. 2009; 73(4):1276-81. doi:10.1016/j.jirobp.2008.10.055.

23. Cattaneo GM, Passoni P, Sangalli G, Slim N, Longobardi B, Mancosu P, et al. Internal target volume defined by contrast-enhanced $4 D-C T$ scan in unresectable pancreatic tumour: evaluation and reproducibility. Radiother Oncol. 2010;97(3):525-9. doi:10.1016/.jradonc.2010.08.007.

24. Taniguchi CM, Murphy JD, Eclov N, Atwood TF, Kielar KN, Christman-Skieller C, et al. Dosimetric analysis of organs at risk during expiratory gating in stereotactic body radiation therapy for pancreatic cancer. Int J Radiat Oncol Biol Phys. 2013;85(4):1090-5. doi:10.1016/j.ijrobp.2012.07.2366.

25. Bert C, Durante M. Motion in radiotherapy: particle therapy. Phys Med Biol. 2011;56(16):R113-44. doi:10.1088/0031-9155/56/16/R01.

26. Liu F, Erickson B, Peng C, Li XA. Characterization and management of interfractional anatomic changes for pancreatic cancer radiotherapy. Int J Radiat Oncol Biol Phys. 2012;83(3):e423-9. doi:10.1016/j.jijrobp.2011.12.073.

27. Batista V, Richter D, Jaekel O, Combs S. OC-0390: Inter-fractional robustness of beam angles and margins in scanned carbon treatment of pancreatic tumors. Radiother Oncol. 2014;111(1):S152-3. doi:10.1016/S0167-8140(15)30495-3.

28. Richter D, Graeff C, Jakel O, Combs SE, Durante M, Bert C. Residual motion mitigation in scanned carbon ion beam therapy of liver tumors using enlarged pencil beam overlap. Radiother Oncol. 2014;113(2):290-5. doi:10. 1016/j.radonc.2014.11.020.

29. Richter D, Saito N, Chaudhri N, Hartig M, Ellerbrock M, Jakel O, et al. Four-dimensional patient dose reconstruction for scanned ion beam therapy of moving liver tumors. Int J Radiat Oncol Biol Phys. 2014;89(1):175-81. doi:10.1016/j.jprobp.2014.01.043.

30. Combs SE, Kalbe A, Nikoghosyan A, Ackermann B, Jakel O, Haberer T, et al. Carbon ion radiotherapy performed as re-irradiation using active beam delivery in patients with tumors of the brain, skull base and sacral region. Radiother Oncol. 2011;98(1):63-7. doi:10.1016/.radonc.2010.10.010.

31. Habermehl D, Wagner M, Ellerbrock M, Buchler MW, Jakel O, Debus J, et al. Reirradiation using carbon ions in patients with locally recurrent rectal cancer at HIT: first results. Ann Surg Oncol. 2014. doi:10.1245/s10434-014-4219-z.

32. Kirkpatrick JP, van der Kogel AJ, Schultheiss TE. Radiation dose-volume effects in the spinal cord. Int J Radiat Oncol Biol Phys. 2010;76(3):S42-9. doi:10.1016/j.jijobp.2009.04.095.

33. Elsasser $T$, Weyrather WK, Friedrich T, Durante M, lancu G, Kramer M, et al. Quantification of the relative biological effectiveness for ion beam radiotherapy: direct experimental comparison of proton and carbon ion beams and a novel approach for treatment planning. Int J Radiat Oncol Biol Phys. 2010;78(4):1177-83. doi:10.1016/j.jirobp.2010.05.014.

34. Scholz M, Kellerer AM, Kraft-Weyrather W, Kraft G. Computation of cell survival in heavy ion beams for therapy. The model and its approximation. Radiat Environ Biophys. 1997;36(1):59-66.

35. Grun R, Friedrich T, Elsasser T, Kramer M, Zink K, Karger CP, et al. Impact of enhancements in the local effect model (LEM) on the predicted RBE-weighted target dose distribution in carbon ion therapy. Physics in medicine and biology. 2012;57(22):7261-74. doi:10.1088/0031-9155/57/22/7261.

\section{Submit your next manuscript to BioMed Central and take full advantage of:}

- Convenient online submission

- Thorough peer review

- No space constraints or color figure charges

- Immediate publication on acceptance

- Inclusion in PubMed, CAS, Scopus and Google Scholar

- Research which is freely available for redistribution

Submit your manuscript at www.biomedcentral.com/submit 